\title{
Rhabdomyosarcome de I'iliopsoas : Localisation Exceptionnelle Chez une Femme de 65 Ans
}

\author{
Koffi-Tessio V.E.S \\ Oniankitan $S$.
}

Centre Hospitalier Universitaire Sylvanus Olympio, Lomé, Togo

Houzou $P$.

Centre Hospitalier Universitaire de Kara, Togo

Kakpovi K.

Centre Hospitalier Régional de Tomdè, Kara, Togo

Fianyo $E$.

Tagbor $K$.

Hôpital de Bè, Lomé, Togo

Oniankitan $O$.

Mijiyawa M.

Centre Hospitalier Universitaire Sylvanus Olympio, Lomé, Togo

Doi:10.19044/esj.2021.v17n14p64

Submitted: 09 December 2020

Accepted: 05 March 2021

Published: 30 April 2021
Copyright 2021 Author(s)

Under Creative Commons BY-NC-ND

4.0 OPEN ACCESS

Cite As:

V.E.S K-T., Oniankitan S., Houzou P., Kakpovi K., Fianyo E., Tagbor K., Oniankitan O. \& Mijiyawa M. (2021). Rhabdomyosarcome de I'iliopsoas : Localisation Exceptionnelle Chez une Femme de 65 Ans. European Scientific Journal, ESJ, 17(14), 64.

https://doi.org/10.19044/esj.2021.v17n14p64

\section{Resume}

Le rhabdomyosarcome est une tumeur maligne mésenchymateuse de l'enfant et de l'adolescent. Il représente 5\% de l'ensemble des tumeurs solides à ces âges. Cette tumeur est rare chez l'adulte et exceptionnelle chez le sujet âgé. Les localisations les plus fréquentes sont la tête, le cou et l'appareil urinaire. Peu d'études rapportent des localisations rétropéritonéales et l'atteinte primitive du muscle iliopsoas a été exceptionnellement décrite dans la littérature. Les auteurs rapportent une nouvelle observation d'une patiente de 65 ans présentant un rhabomyosarcome alvéolaire de l'iliopsoas gauche.

Mots-clés : Iliopsoas, Rhabdomyosarcome, Tumeur, Rétropéritoine 


\title{
Rhabdomyosarcoma of the Iliopsoas: Exceptional Localization in a 65-Year Old Woman
}

\author{
Koffi-Tessio V.E.S \\ Oniankitan $S$. \\ Centre Hospitalier Universitaire Sylvanus Olympio, Lomé, Togo \\ Houzou $\boldsymbol{P}$. \\ Centre Hospitalier Universitaire de Kara, Togo \\ Kakpovi $K$. \\ Centre Hospitalier Régional de Tomdè, Kara, Togo \\ Fianyo $\boldsymbol{E}$. \\ Tagbor $K$. \\ Hôpital de Bè, Lomé, Togo \\ Oniankitan $\boldsymbol{O}$. \\ Mijiyawa $M$. \\ Centre Hospitalier Universitaire Sylvanus Olympio, Lomé, Togo
}

\begin{abstract}
Rhabdomyosarcoma is a malignant mesenchymal tumor in children and adolescents. It represents $5 \%$ of all solid tumors at these ages. This tumor is rare in adults and exceptional in the elderly. The most frequent localizations are the head, the neck, and the urinary tract. Few studies reported retroperitoneal emplacements, and primary involvement of the iliopsoas muscle has been described exceptionally. This paper focuses on reporting a new observation of a 65-year-old patient with alveolar rhabomyosarcoma of the left iliopsoas.
\end{abstract}

Keywords: Iliopsoas, Rhabdomyosarcoma, Tumors, Retroperitoneal

\section{Introduction}

Le rhabdomyosarcome (RMS) est une tumeur maligne d'étiologie indéterminée développée à partir du tissu musculaire strié squelettique caractérisé par son agressivité locorégionale, son évolution métastatique et son pronostic souvent défavorable (Arrob et al., 2018). Il est considéré comme une tumeur de l'enfance et de l'adolescence rarement retrouvée chez l'adulte et les personnes âgées (Desai et al., 2019). Les localisations préférentielles sont respectivement la région de la tête et cou, des voies génito-urinaires et les extrémités ; les localisations moins courantes comprennent le tronc, l'abdomen et surtout la région rétro-péritonéale (Tazi et al., 2000 ; Ognjanovic et al., 2009). Nous rapportons l'observation rarissime d'un RMS de l'iliopsoas chez une patiente de 65 ans vue en rhumatologie. 


\section{Observation}

Il s'agissait d'une patiente de 65 ans admise pour une pygalgie gauche inflammatoire, d'installation progressive avec irradiation à la face antérieure de la cuisse homolatérale et évoluant depuis trois mois. Cette pygalgie devenue invalidante deux semaines avant l'hospitalisation ne s'accompagnait pas d'autres sites douloureux. Cette douleur évoluait dans un contexte fébrile avec amaigrissement sans porte d'entrée infectieuse. Dans ses antécédents, elle a été prise en charge en cardiologie 7 mois avant son admission pour une thrombose veineuse iliaque gauche sans facteur étiologique identifié. L'examen clinique a noté un état général altéré, une fièvre à $39^{\circ} \mathrm{C}$, une attitude du membre inférieur gauche en psoïtis, une palpation douloureuse de la fesse gauche avec signe de Patrick positif. L'examen digestif a révélé à la fosse iliaque gauche, une masse abdominale lisse, ferme, mate et douloureuse. Le rachis, le pelvis et les autres membres étaient sans douleur à la palpation. L'examen spléno-ganglionnaire, thyroïdien, pulmonaire, des seins et urogénital était strictement normal. Les hypothèses de sacroiliite infectieuse et de tumeur osseuse ont été évoquées. L'hémogramme a révélé une anémie microcytaire hypochrome (hémoglobine à $7,5 \mathrm{~g} / \mathrm{dl}$ ) et la vitesse de sédimentation globulaire était de $134 \mathrm{~mm}$ à la première heure. Il existait une hypercalcémie à $118 \mathrm{~g} / \mathrm{l}$, une hypokaliémie à $2,5 \mathrm{meq} / \mathrm{l}$ et une hypochlorémie à $83 \mathrm{mmol} / \mathrm{l}$. La créatininémie était normale à $8 \mathrm{mg} / \mathrm{l}$. Le scanner thoracoabdomo-pelvien a objectivé une formation hypodense de 12 centimètres de diamètre au dépens de l'iliopsoas gauche avec un œdème périlésionnel. Cette lésion est associée à une lyse du sacrum et de l'os iliaque gauche (Figure 1) sans extension au thorax et au reste de l'abdomen. Un examen histopathologique a révélé un RMS alvéolaire de l'iliopsoas gauche (Figure 2). La patiente est décédée des suites des complications des troubles hydroélectrolytiques.

\section{Discussion}

Le RMS est le plus fréquent des sarcomes des tissus mous de l'enfant et de l'adulte jeune. Il représente $6.5 \%$ de toutes les tumeurs malignes en pédiatrie avec une incidence annuelle de 4 à 7 cas/millions d'enfants (Tazi et al., 2000). Il s'agit ainsi d'une tumeur infantile avec une prédilection du type histologique embryonnaire. Il apparaît rarement chez l'adulte et exceptionnellement chez le sujet âgé où le type alvéolaire semble plus fréquent (Van Gaal et al., 2012). C'est le cas de l'observation rapportée qui révèle un RMS alvéolaire chez une femme âgée. En effet, les sites couramment touchés sont la tête et cou, les voies génito-urinaires et les membres (Sultan et al., 2008). Cependant, chez les personnes âgées, une incidence plus élevée est observée dans les sites inhabituels tels que le tronc, la région périnéale et rarement le retropéritoine (Sultan et al., 2008). Sur le plan clinique, notre 
patiente avait un antécédent récent de thrombophlébite gauche inexpliquée ; cette affection cardiovasculaire pouvant parfois être révélatrice d'un RMS du psoas ipsilatéral (Upadhyay et al., 2017); d'où l'intérêt de la recherche rigoureuse et systématique d'un facteur étiologique devant une thrombose veineuse de membre. L'attitude du patient en psoïtis et la présence d'une masse à la fosse iliaque sont des éléments constamment retrouvées dans l'atteinte de l'iliopsoas avec extension locorégionale (Dhaou et al., 2012 ; Upadhyay et al., 2017). Le type histologique alvéolaire est surtout caractérisé par son agressivité régionale avec un risque de métastases (Shahin et al., 2015; Salunke et al., 2017). Le traitement consiste en une résection de la tumeur associée à une chimiothérapie. Généralement, le pronostic est bon pour les patients de moins de 15 ans, mais mauvais pour les adultes avec une mortalité élevée (Salunke et al., 2017).

\section{Conclusion}

Le rhabdomyosarcome de pronostic souvent sévère, bien que rare chez l'adulte et de localisation exceptionnelle à l'iliopsoas, ne doit pas être méconnu en cas d'une tumeur rétropéritonéale avec attitude en psoïtis chez un sujet âgé.
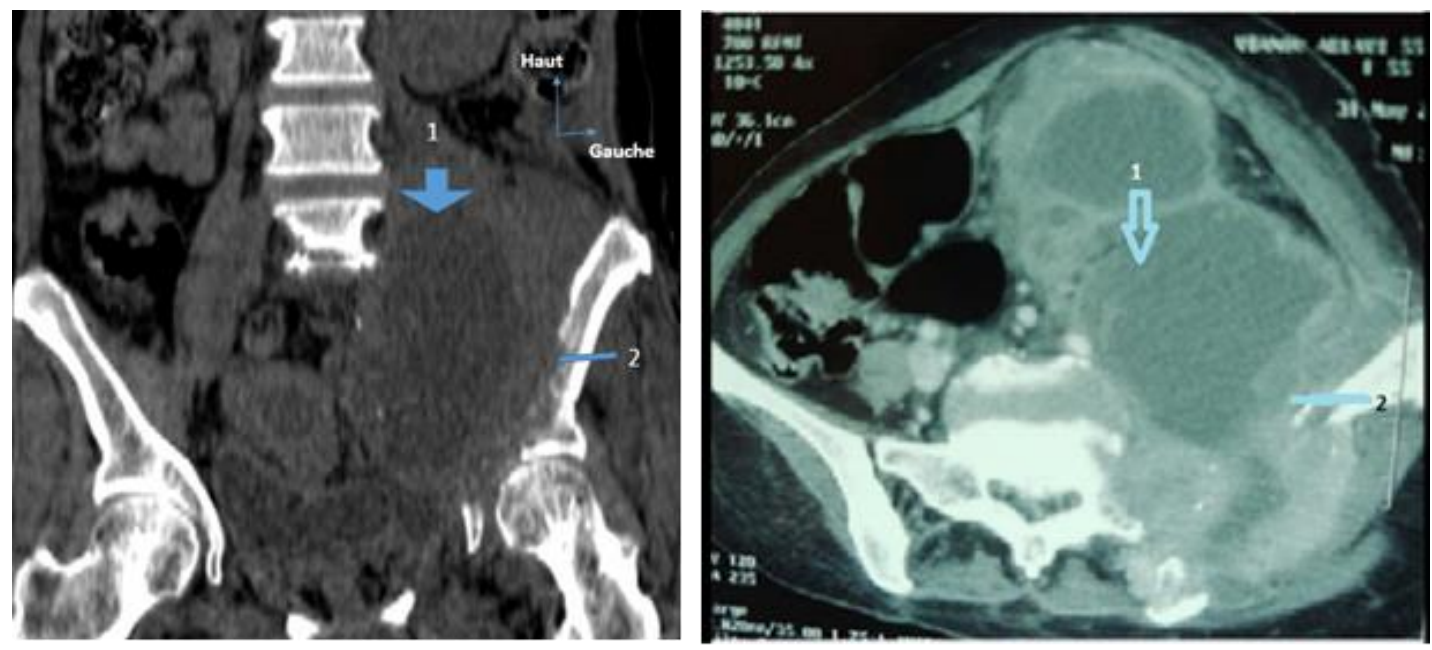

Figure 1. Masse hypodense développée au dépens de l'iliopsoas gauche (1) associée à une lyse du sacrum et de l'os iliaque (2) 


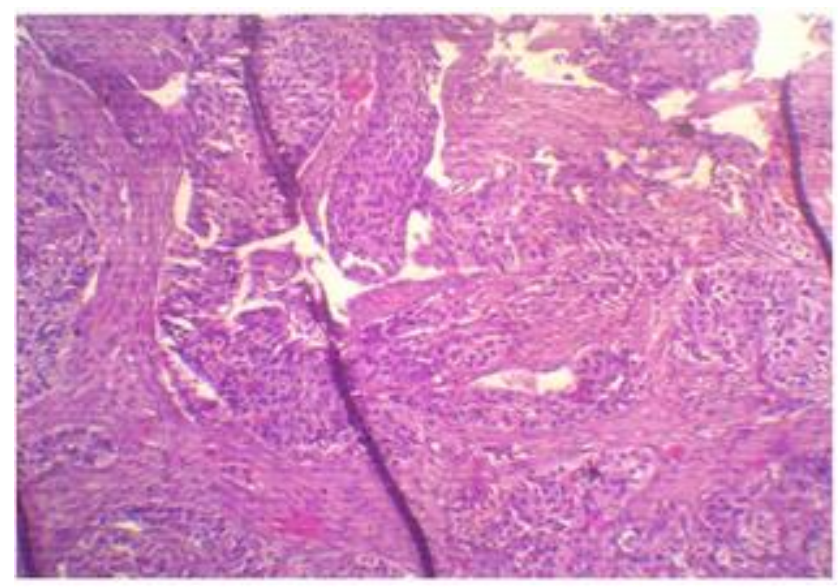

Figure 2. Aspect histologique du rhabdomyosarcome alvéolaire

\section{References :}

1. Arrob, A., Fiqhi, MK., N'diaye, A., El Khatib, K. \& Abouchadi, A. (2018). Rhabdomyosarcome cranio-facial de l'adulte : un cas de mauvais pronostic. PAMJ;30:3.

2. Desai, KB., Mella, D., \& Pan, E. (2019). An Adult Patient With Rare Primary Intracranial Alveolar Rhabdomyosarcoma. Anticancer Res. 2019;39:3067-70.

3. Dhaou, B., Aydi, Z., Baili, L., Boussema, F., Rokbani, L. (2012). Phlébite du membre inférieur révélant un rhabdomyosarcome du psoas chez une femme âgée. Rev francoph gériatr gérontol;19 : 237-238.

4. Ognjanovic, S., Linabery, AM., Charbonneau, B., \& Ross, JA. (2009). Trends in childhood rhabdomyosarcoma incidence and survival in the United States, 1975-2005. Cancer;115:4218-26.

5. Salunke, AA., Shah, J., Gupta, N., \& Pandit, J. (2017). Adult rhabdomyosarcoma: Multimodality management and results. J Cancer Res Ther; 13:384.

6. Shahin, NA., Alqaisy, A., \& Zheng, W. (2015). Primary alveolar rhabdomyosarcoma of fallopian tube masquerading as a unilateral adnexal mass: A case report and literature review. Indian J Pathol Microbiol. déc 2015;58:521-3.

7. Sultan, I., Qaddoumi, I., Yaser, S., Rodriguez-Galindo, C., \& Ferrari, A. (2009). Comparing adult and pediatric rhabdomyosarcoma in the surveillance, epidemiology and end results program, 1973 to 2005: An analysis of 2,600 patients. J Clin Oncol; 27:3391-7.

8. Tazi, K., Moudouni, S., Koutani, A., Ibn Attya, A., Hachimi, M. \& Lakrissa, A. (2000). Rabdomyosarcome paratesticulaire de l'adulte jeune. Prog Urol, 2000;10:469-472. 
9. Upadhyay, AA. (2017). Rhabdomyosarcoma of the Iliopsoas: A Retroperitoneal Misdiagnosis. Indian J Med Paediatr Oncol. 2017 JulSep; 38: 401-403.

10. Van Gaal, JC., Van Der Graaf, WTA., Rikhof, B., \& Van Hoesel, QGCM., Teerenstra, S., Suurmeijer, AJH, et al. (2012). The impact of age on outcome of embryonal and alveolar rhabdomyosarcoma patients. A multicenter study. Anticancer Res Oct;32:4485-97. 